\title{
Role of Rho kinase in lysophosphatidic acid-induced altering of blood-brain barrier permeability
}

\author{
YING YU $^{1}$, JUN QIN ${ }^{2}$, MEIZHEN LIU $^{1}$, QINGYUAN RUAN ${ }^{1}$, YILIANG LI ${ }^{1}$ and ZHAOHUI ZHANG ${ }^{1}$ \\ ${ }^{1}$ Department of Neurology, Renmin Hospital of Wuhan University, Wuhan, Hubei 430060; \\ ${ }^{2}$ Department of Orthopaedic Surgery, Zhongnan Hospital of Wuhan University, Wuhan, Hubei 430071, P.R. China
}

Received October 4, 2013; Accepted December 27, 2013

DOI: $10.3892 / \mathrm{ijmm} .2014 .1618$

\begin{abstract}
Lysophosphatidic acid (LPA) the simplest of the water-soluble phospholipids, is produced by activated platelets, macrophage and endothelial cells. It also evokes various biological responses. When LPA concentrations reach high levels, brain injury, including stroke and intracerebral hemorrhage (ICH), occurs. Previous studies have shown that LPA is crucial in increasing blood-brain barrier (BBB) permeability, and the Rho/Rho kinase (ROCK) signaling pathway is involved in the regulation of endothelial permeability. However, the exact mechanism by which the Rho/ROCK pathway mediates BBB disruption induced by LPA remains to be determined. In the present study, we observed that LPA induced the increase of BBB permeability in the right striatum after $10 \mu \mathrm{l}$ LPA $(100 \mu \mathrm{M})$ was injected into the ipsilateral caudate nucleus of rats. The ROCK was involved in the expression of proteolytic enzymes, matrix metalloproteinase (MMP)-9 and urokinase-type plasminogen activator (uPA), leading to LPA-induced BBB disruption. ROCK inhibitor (Y27632) markedly inhibited the expression of proteolytic enzymes induced by LPA as well as the BBB disruption after it was co-injected with LPA. Thus, results of the present study suggest that LPA increases BBB permeability, which may be due to the Rho/ROCK signaling pathway and the subsequent production of proteolytic enzymes MMP-9 and uPA.
\end{abstract}

\section{Introduction}

Lysophosphatidic acid (LPA), which is released from activated platelets (1) and the hydrolysis of circulating lysophospholipids (2), is a simple phospholipid that is involved in a number of cell processes including proliferation, migration, platelet

Correspondence to: Professor Zhaohui Zhang, Department of Neurology, Renmin Hospital of Wuhan University, 238 Jiefang Road, Wuhan, Hubei 430060, P.R. China

E-mail: zhangzhaohui18@hotmail.com

Key words: lysophosphatidic acid, blood-brain barrier, Rho kinase, matrix metalloproteinase-9, urokinase-type plasminogen activator aggregation, neurite retraction and neuropathic pain (1,3-6). LPA is detected in serum, plasma, other biological fluids, and tissues including brain (3). It is produced in the course of blood coagulation in humans (7). Levels of LPA are increased after brain injury, including intracerebral hemorrhage (ICH) (8) and cerebral ischemia (CI) (9). LPA is also produced by activated platelets and stimulates platelet aggregation in turn, forming a positive feedback and causing significantly elevated levels of LPA in a local region of the brain, resulting in clotting hematoma in ICH or thrombosis in CI.

Blood-brain barrier (BBB) disruption and brain edema is an important pathophysiological event of brain injury after ICH or CI. Proteolytic enzymes and inflammatory cytokines change the BBB permeability properties, leading to brain edema formation. Various proteolytic enzymes, including matrix metalloproteinase (MMP)-9 and urokinase-type plasminogen activator (UPA) have been suggested to be critical mediators for altering BBB permeability (10). MMP-9 degrades the basement membrane, and uPA stimulates the production of plasmin that degrades various components of extracellular matrix. Previous studies have indicated that LPA is involved in increasing endothelial monolayer permeability (11), and that a high concentration of LPA may disrupt the BBB (8). However, the exact molecular mechanism that serves as a contributory factor of LPA to BBB disruption remains to be determined.

The Rho/Rho kinase (ROCK) signaling pathway is important in the regulation of endothelial permeability. ROCK, the downstream effector of Rho, is a serine/threonine kinase that is activated by binding to the active GTP-bound form of Rho. Results of recent studies have demonstrated that ROCK increases BBB permeability via the upregulation of MMP-9 and disruption of tight junction proteins $(12,13)$. The ROCK inhibitor prevents injury to brain endothelial cells by the reduction of MMP-9 activity (14). Mounting evidence supports that the Rho/ROCK signaling pathway is crucial in cancer cell migration and invasion induced by LPA, which is due to the upregulation of proteolytic enzyme secretion by Rho/ROCK signaling pathway (15). Considering that LPA is capable of increasing BBB permeability, and that the Rho/ROCK pathway plays a role in LPA-induced proteolytic enzymes secretion, including uPA and MMPs, we hypothesized that LPA-induced ROCK activation is involved in proteolytic enzyme secretion, leading to the disruption of BBB in vivo. 
The aim of the present study was to examine whether LPA induces the secretion of proteolytic enzymes MMP-9 and $\mathrm{UPA}$, to increase the permeability of BBB in vivo, and whether ROCK is critical for LPA-induced proteolytic enzyme expression as well as BBB disruption. The results obtained suggest mechanisms by which LPA increases permeability of BBB through proteolytic enzyme secretion mediated by a Rho/ROCK signaling pathway.

\section{Materials and methods}

Animals. Adult male Sprague-Dawley rats (weight, 250-280 g) obtained from the Animal Center of Wuhan University were housed under controlled temperature and lighting conditions with food and water. All animal procedures were reviewed and approved by the international guidelines for the ethical use of laboratory animals and the National Institutes of Health Guide for the Care and Use of Laboratory Animals.

Surgical procedure. Animals were anesthetized by intraperitoneal injection of ketamine $(60 \mathrm{mg} / \mathrm{kg})$ and xylazine $(10 \mathrm{mg} / \mathrm{kg})$, and then placed in a stereotaxic frame (Bilaney Consultants, Düsseldorf, Germany). A scalpel was used to expose the skull, and a hole was drilled at $3.0 \mathrm{~mm}$ laterally and $0.2 \mathrm{~mm}$ anterior to the bregma. A microinjection needle was inserted stereotaxically into the right caudate nucleus (coordinates: $0.2 \mathrm{~mm}$ anterior, $5.5 \mathrm{~mm}$ ventral and $3.0 \mathrm{~mm}$ lateral to the bregma). LPA (Sigma, St. Louis, MO, USA) was dissolved in sterile phosphate-buffered saline (PBS) and a final concentration of $100 \mu \mathrm{M}$ was delivered in a final volume of $10 \mu \mathrm{l}$ over $5 \mathrm{~min}$; the needle remained in place for an additional $5 \mathrm{~min}$ to prevent reflux. ROCK inhibitor Y27632 (Sigma) was dissolved in sterile PBS at a final concentration of $1 \mathrm{mM}$ and co-injected with LPA into the right caudate nucleus in some experiments to detect whether Y27632 decreased BBB permeability by inhibiting ROCK. The hole in the skull was sealed with bone wax and the scalp was sutured. Control rats were infused with the same volume of PBS, and the brains were removed $24 \mathrm{~h}$ after microinjection. Rectal temperature was monitored and maintained between 36.5 and $37^{\circ} \mathrm{C}$ with a heating pad throughout the procedure. The overall mortality rate was $<1 \%$. Striatum tissues in each group were obtained as described in Fig. 1 for subsequent detection.

Experimental groups. Animals (150 rats) were randomly assigned to each group. Six rats used for the ultrastructural analysis of BBB were sacrificed $24 \mathrm{~h}$ after surgery as well as controls ( $n=3$ for each group). Thirty-six rats used for determination of extravasated Evans blue dye (EBD) for BBB integrity; 36 rats for quantitative polymerase chain reaction (qPCR); 36 rats for western blot detection; 36 rats for the immunohistochemistry evaluation ( $\mathrm{n}=6$ for each time-point at 6, 24, 48 and $72 \mathrm{~h}$ after LPA was intracerebrally injected and $24 \mathrm{~h}$ after LPA co-injected with Y27632 as well as for controls).

Evans blue dye extravasation. BBB permeability was quantitatively evaluated by the fluorescent detection of extravasated EBD at 6, 24, 48 and $72 \mathrm{~h}$ after injection of LPA into the right caudate nucleus of rats (16). Briefly, 2\% EBD in saline was injected from tail vein ( $4 \mathrm{ml} / \mathrm{kg}$; Sigma) and allowed to circulate for $60 \mathrm{~min}$. To remove the intravascular dye, the animals were perfused with saline through the left ventricle at $100 \mathrm{~cm} \mathrm{H}_{2} \mathrm{O}$ until colorless perfusion fluid was obtained from the right atrium. After rats were decapitated, four sections of the brains were removed: ipsilateral striatum and cortex, and contralateral striatum and cortex (data not shown). Each tissue sample was weighed, homogenized in $2 \mathrm{ml}$ of $50 \%$ trichloroacetic acid (wt/vol), and centrifuged at $18,000 \mathrm{x} \mathrm{g}$ for $20 \mathrm{~min}$. EBD was extracted from the tissue by using $50 \%$ trichloroacetic acid to dissociate the dye from protein. Subsequent to centrifugation, the supernatants containing EBD were diluted 4-fold with ethanol. For fluorescence measurement, an aliquot was diluted with solvent (50\% trichloroacetic acid:ethanol =1:3). Tissue levels of EBD were quantified by using a spectrofluorometer at an excitation wavelength of $620 \mathrm{~nm}$ and an emission wavelength of $680 \mathrm{~nm}$. Sample values were compared with those of EBD standards mixed with the solvent (100-1,000 $\mu \mathrm{g} / \mathrm{l})$. The EBD contents were expressed as $\mu \mathrm{g} / \mathrm{g}$ brain weight.

Ultrastructural analysis. Rats were perfused through the left ventricle with saline followed by $2.5 \%$ glutaraldehyde and $2 \%$ paraformaldehyde in $0.1 \mathrm{M}$ cacodylate buffer at $\mathrm{pH} 7.4$. Tissue sections from the striatum were additionally fixed in the same fixative for $2 \mathrm{~h}$ at $4^{\circ} \mathrm{C}$. After washing, the slices were post-fixed in $1 \% \mathrm{OsO}_{4}$ for $1 \mathrm{~h}$. The slices were subsequently dehydrated in graded ethanol, and embedded in Epon 812. For electron microscopy, ultrathin sections were processed by cutting with a diamond knife on a Ultramicrotome (Leica Ultracut UCT; Leica Microsystems GmbH, Wetzlar, Germany) and then collected on copper grids. The material was air-dried, then stained for 10 min with $4.7 \%$ uranyl acetate and for $2 \mathrm{~min}$ with lead citrate. The sections were examined and images were captured on a Philips G212 electron microscope (FEI Tecnai; FEI, Amsterdam, The Netherlands).

$q P C R$. Animals were sacrificed at 6, 24, 48 and $72 \mathrm{~h}$ following LPA injection into the right caudate nucleus. Total RNA was extracted from the right striatum tissues using TRIzol (Invitrogen, Carlsbad, CA, USA) following the manufacturer's instructions. The concentration and purity were measured using the NanoDrop ${ }^{\mathrm{TM}}$ method (3300 NanoDrop Analyzer; Thermo Scientific, Foster City, CA, USA). Equal amounts $(1 \mu \mathrm{g})$ of total RNA were reverse transcribed into cDNA using a first-strand cDNA synthesis kit (Transgen Biotech Co., Ltd., Beijing, China). PCR was performed with primers for MMP-9, forward: 5'-ACGAGGACTCCCCTCTGCAT-3' and reverse: 5'-AGGCCTTGGGTCAGGTTTAGA-3'; uPA, forward: 5'-ACAGATTCCTGCTCGGGAGAT-3' and reverse: 5'-CCAATGTGGGACTGAATCCAG-3'; ROCK2 forward: 5'-AACCTACTCCTGGAAGCCG-3' and reverse: 5'-AGACAACGCTTCTGAGTTTCC-3'; glyceraldehyde 3-phosphate dehydrogenase (GAPDH), forward: 5'-CAGT GCCAGCCTCGTCTCAT-3' and reverse: 5'-TGGTAACCA GGCGTCCGATA-3'). Following an initial incubation for $15 \mathrm{~min}$ at $95^{\circ} \mathrm{C}$, the reactions were carried out for 40 cycles at $95^{\circ} \mathrm{C}$ for $15 \mathrm{sec}$ and $60^{\circ} \mathrm{C}$ for $30 \mathrm{sec}$ (florescence collection). The expression of MMP-9, uPA and ROCK2 was normalized to GAPDH gene and compared with the control. 

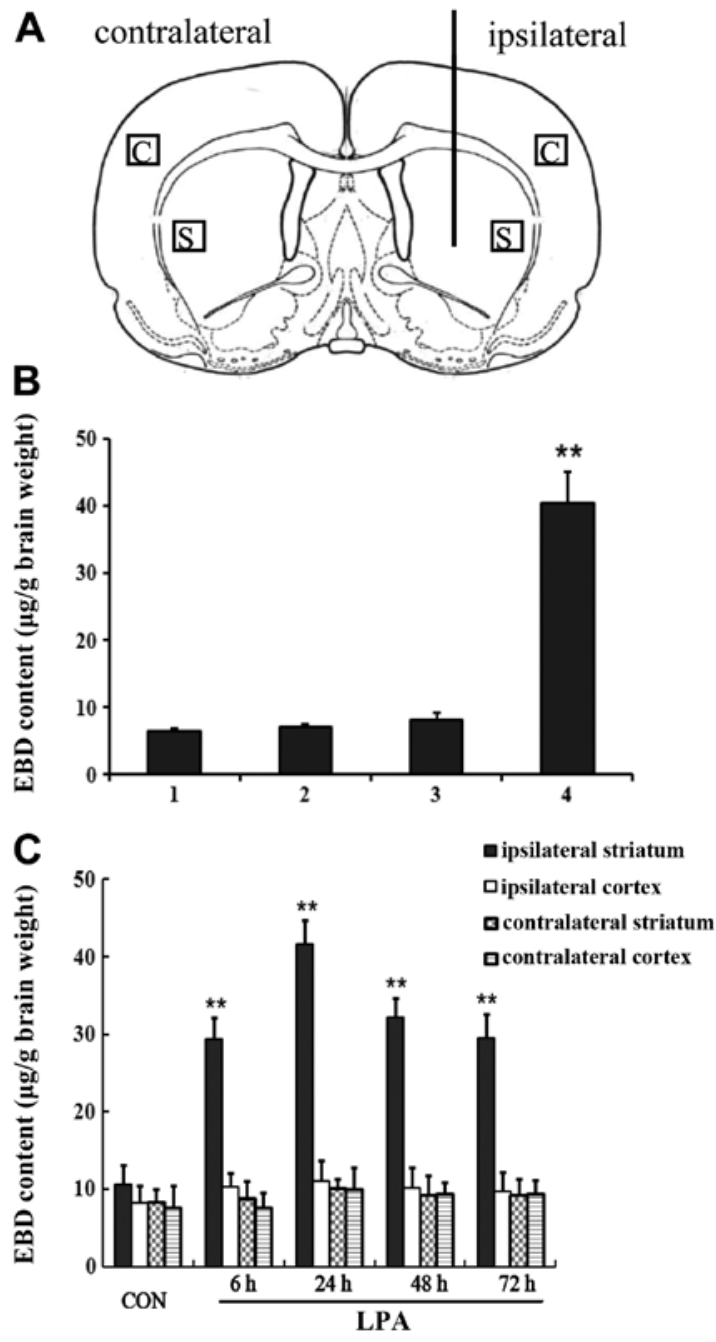

Figure 1. Effect of lysophosphatidic acid (LPA) on the permeability of blood-brain barrier (BBB). (A) Four sections of the brain were removed for Evans blue dye (EBD) content detection: ipsilateral striatum (S) and cortex (C), and contralateral striatum (S) and cortex (C). (B) The EBD content in the ipsilateral striatum was detected $24 \mathrm{~h}$ after the solution was injected into the right caudate nucleus of rats in the following four groups: 1 , the sham operation group: the skull was exposed and a hole was drilled, then the scalp was sutured; 2 , the needle-inserted group: the needle was inserted stereotaxically into the right caudate nucleus but no solution was injected; 3 , the control group: $10 \mu 1$ phosphate-buffered saline (PBS) was injected into the right caudate nucleus; 4 , the LPA group: $10 \mu 1 \mathrm{LPA}$ $(100 \mu \mathrm{M})$ was injected into the right caudate nucleus. (C) The EBD content was detected in the different brain regions as indicated in (A) at $6,24,48$ and $72 \mathrm{~h}$ in the LPA injection and control groups. ${ }^{* *} \mathrm{P}<0.01$ vs. the control.

Western blotting. The brains of rat were removed at 6,24,48 and $72 \mathrm{~h}$ after LPA injection, and the right striatum was dissected, immediately frozen and kept at $-70^{\circ} \mathrm{C}$. The frozen tissue was homogenized in homogenization buffer $(\mathrm{pH} 7.4)$ with protease inhibitors (Sigma) and centrifuged at 12,000 $\mathrm{x}$ g for $20 \mathrm{~min}$ at $4^{\circ} \mathrm{C}$. The supernatant was then collected and the total protein was determined using a Micro BCA Protein Assay kit. Protein $(50 \mu \mathrm{g})$ of each sample was loaded onto the $8-10 \%$ SDS polyacrylamide gel (SDS-PAGE), separated by electrophoresis and transferred to nitrocellulose membranes (EMD Millipore Co., Billerica, MA, USA). The membranes were incubated at $4^{\circ} \mathrm{C}$ overnight with primary antibodies: anti-MMP-9 (goat polyclonal, 1:400), anti-uPA (rabbit polyclonal, 1:400), antiROCK2 (goat polyclonal, 1:100) and anti- $\beta$-actin (mouse monoclonal, 1:500), all from Santa Cruz Biotechnology, Inc. (Santa Cruz, CA, USA). After being washed three times for 10 min with washing solution, the membranes were incubated with horseradish peroxidase-conjugated secondary antibodies (1:1,000; ZSGB-Bio, Beijing, China). Immunoreactive bands were visualized by an enhanced chemiluminescent substrate kit and exposure to CL-XPosure film. The immunoreactivity of proteins bands was quantitatively analyzed by Kodak Digital Science 1D software (Eastman Kodak Co., Rochester, NY, USA) and expressed as a mean optical density. Relative levels of MMP-9, uPA and ROCK2 protein were normalized to $\beta$-actin and compared with the control.

Immunohistochemical staining. To assess the spatial distribution of MMP-9 and uPA after LPA injection, immunohistochemistry was performed. Briefly, the brain was dehydrated and embedded in paraffin. Then it was sliced coronally into $4 \mu \mathrm{m}$ sections from the rostral to the caudal section of the injection site. The sections were de-waxed and rehydrated, rinsed with distilled water and PBS, quenched with $3 \% \mathrm{H}_{2} \mathrm{O}_{2}$, blocked in 10\% normal goat serum, and incubated overnight at $4^{\circ} \mathrm{C}$ with primary antibodies: anti-MMP-9 (goat polyclonal, 1:100) or anti-uPA (rabbit polyclonal, 1:100) both from Santa Cruz Biotechnology, Inc. The sections were then incubated with biotinylated goat anti-mouse IgG or rabbit anti-goat IgG (1:400; ZSGB-Bio) for $1 \mathrm{~h}$ at room temperature and incubated with streptavidin-peroxidase for $30 \mathrm{~min}$. The immunoreactions were visualized with diaminobenzidine- $\mathrm{H}_{2} \mathrm{O}_{2}$ solution. Sections were washed, successively dehydrated in ethanol, and defatted in xylenes. For the negative controls we used non-specific IgG instead of the primary antibodies. To quantify the number of immunoreactive cells labeled with MMP-9 or uPA, two sections per rat were selected, and five randomly selected non-overlapping high-power fields were examined from each section.

Statistical analysis. Data were expressed as the means \pm SEM. Statistical analysis of quantified data was performed by analysis of variance (ANOVA). $\mathrm{P}<0.05$ was considered to indicate a statistically significant difference.

\section{Results}

$L P A$ increased the permeability of $B B B$ in ipsilateral striatum. To study the effect of LPA on the permeability of BBB, it was injected directly into the rat brain. The extravasation of EBD, as a marker of BBB breakdown, was quantified by spectrofluorophotometric analysis. Since the striatum tissues for the subsequent detection was obtained at a distance from the injection site (Figs. 1A and 2A), the needle itself and the volume of injected solution had no effect on the permeability of BBB (Fig. 1B). No significant difference on the extravasation of EBD in the ipsilateral cortex, contralateral striatum and contralateral cortex between LPA-injected rats and control rats was observed (Fig. 1C). Quantitative analysis of EBD in another three regions demonstrated that leakage within the ipsilateral striatum regions following LPA injection was highest among the ipsilateral cortex and contralateral brain regions, including the contralateral striatum and contralateral cortex (Fig. 1C). We found that the intracerebral injection of 

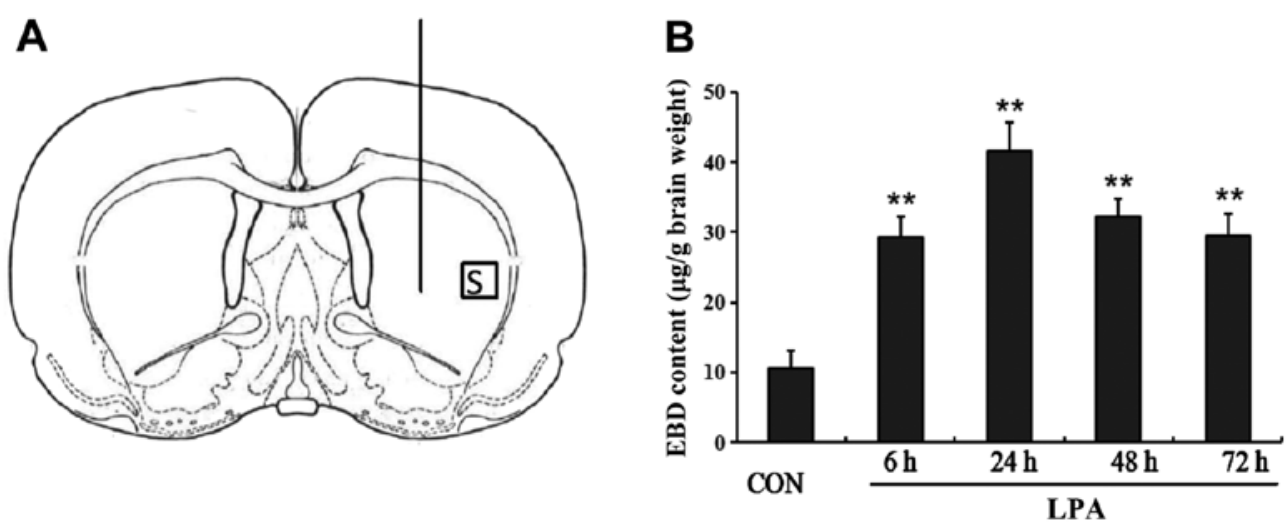

Figure 2. Effect of lysophosphatidic acid (LPA) on the permeability of blood-brain barrier (BBB) in the ipsilateral striatum of rats. (A) Striatum tissues (S) used in our experiment were obtained from a distance from the injection site. (B) The Evans blue dye (EBD) content in the ipsilateral striatum at 6, 24, 48 and $72 \mathrm{~h}$ following LPA injection as well as the control group. ${ }^{* *} \mathrm{P}<0.01 \mathrm{vs}$. the control.
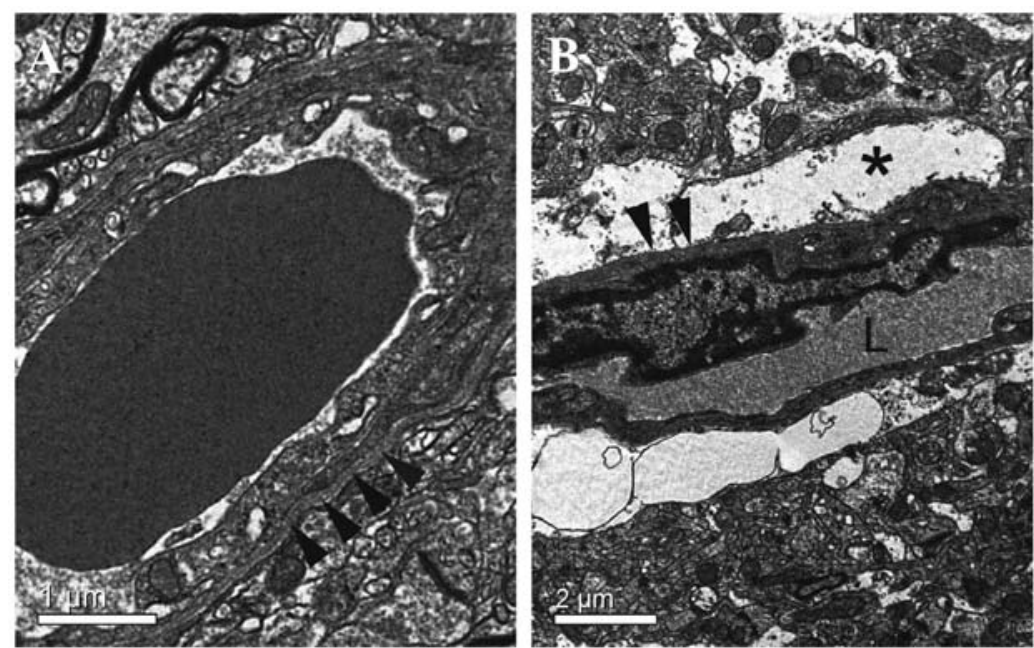

Figure 3. The BBB ultrastructure alterations in the ipsilateral striatum at $24 \mathrm{~h}$ after lysophosphatidic acid (LPA) injection. (A) The regular capillary lumen, integrity base membrane (arrow head), and normal extracellular space were observed in the control group. Scale bar: $1 \mu \mathrm{m}$. (B) The swelling of astrocytic end-feet (asterisk), disrupted base membrane (arrow head), narrowed capillary lumen (L) and enlarged extracellular space were observed at $24 \mathrm{~h}$ after LPA injection. Scale bar, $2 \mu \mathrm{m}$.

LPA resulted in an increase in the permeability of BBB over time. LPA induced an increase in EBD extravasation in the injected striatum, which began to increase $6 \mathrm{~h}$ after LPA injection and peaked at $24 \mathrm{~h}$, whereas it decreased at 48 and $72 \mathrm{~h}$ after LPA was injected intracerebtally (Fig. 2B). Significant differences were noted in the extravasation of EBD in the ipsilateral striatum from 6 to $72 \mathrm{~h}$ after LPA injection compared with controls $(\mathrm{P}<0.01)$ (Fig. 2B).

$B B B$ ultrastructure alterations. Ultrastructural alterations of BBB were studied by transmission electron microscopy in the ipsilateral striatum. According to the result of EBD extravasation detection, the detection time-point was $24 \mathrm{~h}$ after LPA injection when the BBB permeability reached the peak point induced by LPA. Ultrastructural analysis revealed no abnormalities in any structural or cellular elements of the BBB in the control group (Fig. 3A). At $24 \mathrm{~h}$ after LPA was injected intracerebrally, disruption of the BBB was evident. Characteristic large spaces between capillaries and neuropil were observed. In such capillaries, endothelial cells had little alteration in their appearance and internal structure, although their pinocytic activity was increased. The basement membrane was disrupted and extremely coarse. Large swollen astrocytes or astrocytic end-feet with scarce organelles and glycogen particles were observed, and the extracellular space appeared considerably enlarged with the presence of edema fluid, which narrowed capillary lumen (Fig. 3B).

LPA induced the expression of proteolytic enzymes. MMPs and uPA are important enzymes involved in BBB damage, thus we determined the effects of LPA on the expression of these proteolytic enzymes. According to the results of qPCR, LPA significantly increased the mRNA expression of MMP-9 and uPA in the ipsilateral striatum (Fig. 4A). The mRNA expression of MMP-9 and uPA showed a significant difference from 6 to $72 \mathrm{~h}$ following LPA injection as compared to the controls $(\mathrm{P}<0.01)$ (Fig. 4A). The time course of MMP-9 and UPA protein expression assessed by western blotting demonstrated that they were detectable at $6 \mathrm{~h}$ following LPA injection and maximally expressed at $24 \mathrm{~h}$ after LPA injection (Fig. 4B-D). The protein 

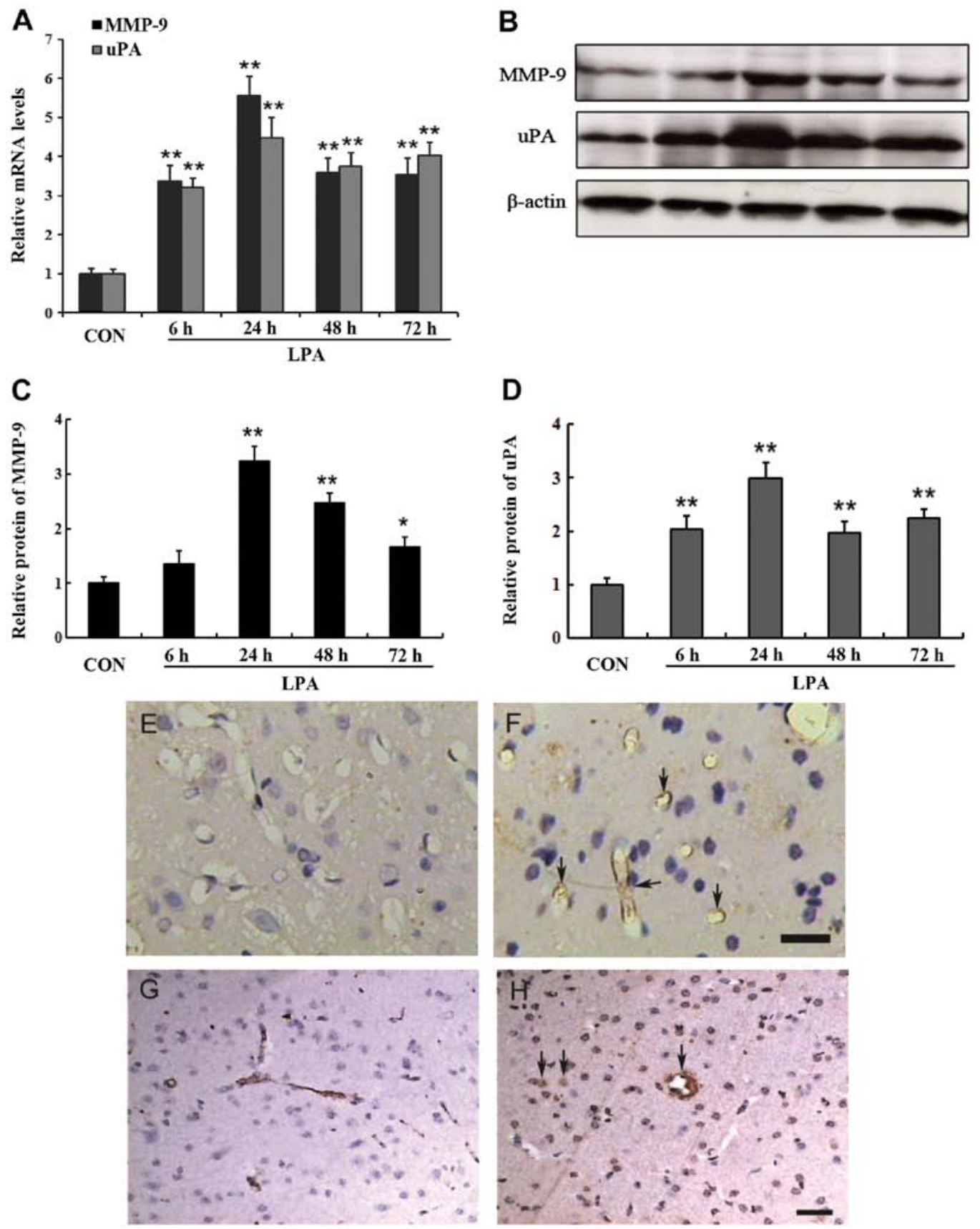

Figure 4. The expression of matrix metalloproteinase (MMP)-9 and urokinase-type plasminogen activator (uPA) induced by lysophosphatidic acid (LPA) at different time-points. (A) The mRNA expression of MMP-9 and uPA in the ipsilateral striatum at 6,24,48 and $72 \mathrm{~h}$ after LPA injection as well as controls. Relative mRNA levels of MMP-9 and uPA normalized to glyceraldehyde 3-phosphate dehydrogenase (GAPDH) and were compared with controls. (B-D) A representative western blot and quantitative results for MMP-9 and uPA protein expression are shown for the analysis at 6, 24, 48 and $72 \mathrm{~h}$ following LPA injection in the ipsilateral striatum as well as the controls. Relative protein levels of MMP-9 and uPA were normalized to $\beta$-actin and compared with controls. (E and F) The protein expression of MMP-9 was detected by immunohistochemical staining. (E) No labeling was identified in the ipsilateral striatum after phosphate-buffered saline (PBS) injection in the control group. (F) The expression of MMP-9 in capillary endothelial cells in the ipsilateral striatum was detected by immunohistochemistry staining at $24 \mathrm{~h}$ after LPA injection. MMP-9 is mainly located on the capillary as a ring or linear brown stains (arrow). Scale bar, $25 \mu \mathrm{m}$. (G and H) The protein expression of uPA detected by immunohistochemical staining. (G) uPA was slightly stained mainly on the endothelial cells of microvessels in the control group. $(\mathrm{H})$ Immunoreactive uPA is observed within endothelial cells and fibrin deposition of microvessels, damaged neurons and glial cells in the ipsilateral striatum at $24 \mathrm{~h}$ after LPA injection (arrow). Scale bar, $25 \mu \mathrm{m}$. ${ }^{*} \mathrm{P}<0.05$ and ${ }^{* *} \mathrm{P}<0.01 \mathrm{vs}$. the control group.

expression of MMP-9 and uPA showed a statistical difference from 24 to $72 \mathrm{~h}$ following LPA injection compared with the controls $(\mathrm{P}<0.05)$ (Fig. 4C and $\mathrm{D})$. The spatial distribution of MMP-9 induced by LPA was evaluated by immunohistochemical analysis. Diffuse MMP-9 expression was observed in the right striatum where LPA was injected. Immunoreactive MMP-9 appeared to stain with the endothelial cells of microvessels (identified by morphometric criteria) at $6,24,48$ and $72 \mathrm{~h}$ after LPA injection (Fig. 4F). By contrast, there was no similar labeling of endothelial cells in the brain from rats that had been injected PBS as controls (Fig. 4E). The MMP-9 expression appeared to present with a significant number of endothelial cells $24 \mathrm{~h}$ after LPA injection. It was consistent with the results obtained from the western blot analysis. The 

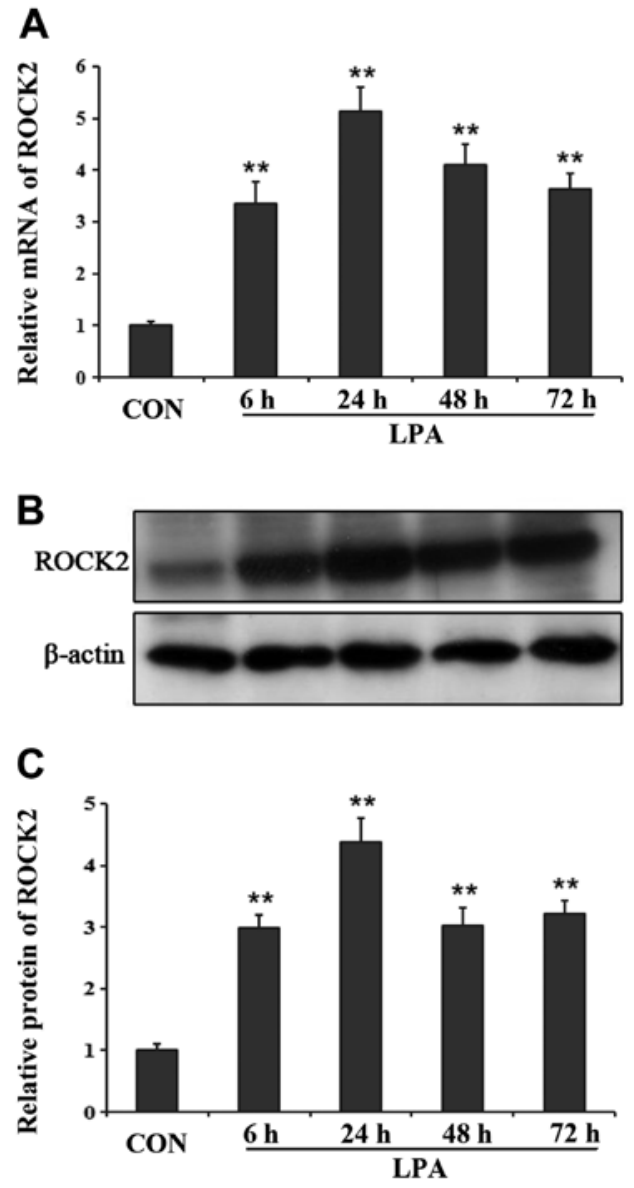

Figure 5. The expression of Rho/Rho kinase (ROCK)2 induced by lysophosphatidic acid (LPA) at different time-points. (A) LPA induces the mRNA expression of ROCK2 in the ipsilateral striatum at 6, 24, 48 and $72 \mathrm{~h}$ after LPA injection as well as controls. The relative mRNA levels of the matrix metalloproteinase (MMP)-9 and urokinase-type plasminogen activator (uPA) normalized to glyceraldehyde 3-phosphate dehydrogenase (GAPDH) compared with the control group. (B and C) A representative western blot and quantitative results for the protein expression of ROCK2 are showsn for the analysis at 6,24, 48 and $72 \mathrm{~h}$ after LPA was injected in the ipsilateral striatum and controls. The relative protein levels of ROCK2 normalized to $\beta$-actin compared with the controls. ${ }^{*} \mathrm{P}<0.05$ and ${ }^{* *} \mathrm{P}<0.01$ vs. the control group.

location of uPA protein induced by LPA was also detected by immunohistochemistry. In the LPA-treated groups, we observed that the staining for uPA protein was predominantly associated with the microvessels and parenchyma and diffusely within regions of the ipsilateral striatum. Immunoreactive uPA was observed within endothelial cells and fibrin deposition of microvessels, damaged neurons and glial cells (identified by morphometric criteria), as well as scattered accumulations within the extracellular space (Fig. 4H). In the control group, uPA was slightly stained mainly on the endothelial cells of the microvessels. No apparent staining of uPA was evident in the neurons or glial cells in the ipsilateral striatum (Fig. 4G). Increased uPA immunoreactivity was noted at 6,24,48 and $72 \mathrm{~h}$ following LPA injection, compared with the PBS-injected controls. The expression of uPA protein was initally detected in the ipsilateral striatum at $6 \mathrm{~h}$ after LPA intracerebral injection, and became apparent at $24 \mathrm{~h}$, then decreased at $48 \mathrm{~h}$, consistent with the results of uPA expression detected by western blotting.
ROCK2 was involved in BBB disruption induced by LPA. ROCK 2 preferentially expressed in the brain and the skeletal muscle (17), thus ROCK 2 was examined in our experiment. By qPCR, we observed the changes of the mRNA level of ROCK2 at different time-points following LPA injection into the right striatum of rats. The expression level of ROCK2 increased at 6 and $24 \mathrm{~h}$ following LPA injection, then decreased gradually at 48 and $72 \mathrm{~h}$ after LPA injection (Fig. 5A). However, the different time-point groups following LPA intracerebral injection all showed a significant difference in the mRNA expression of ROCK2 compared with the control group $(\mathrm{P}<0.01)$. Results of the western blot analysis revealed similar changes in the protein expression of ROCK2 at each timepoint after LPA injection. At $24 \mathrm{~h}$ after LPA was injected into the right striatum, the protein expression of ROCK2 reached a peak point and began to decrease at $48 \mathrm{~h}$ after LPA injection (Fig. 5B and C). The differences were significant between the LPA- and PBS-injected groups $(\mathrm{P}<0.01)$.

ROCK inhibitor decreased BBB permeability and proteolytic enzyme expression. Since ROCK played a critical role in the permeability of the rat BBB in certain pathological conditions $(13,18)$, the ROCK specific inhibitor Y27632 was utilized to evaluate its effects on the increased BBB permeability induced by LPA. We examined the impact of Y27632 on BBB permeability by measuring the EBD content in the ipsilateral striatum at $24 \mathrm{~h}$ after LPA was co-injected with Y27632 into the right caudate nucleus. Compared with the controls, EBD extravasation in the ipsilateral striatum was significantly higher in both $24 \mathrm{~h}$ after LPA injection and LPA co-injection with Y27632 (P<0.01) (Fig. 6A). However, EBD extravasation was significantly lower at $24 \mathrm{~h}$ following LPA co-injection with Y27632 than at the same time-point after LPA injection $(\mathrm{P}<0.05)$ (Fig. 6A). We also examined the effect of Y27632 on the mRNA and protein expression of MMP-9 and uPA in the ipsilateral striatum after LPA co-injection. The results of qPCR and western blot analysis co-demonstrated that the mRNA and protein level of MMP-9 and uPA were higher in both at $24 \mathrm{~h}$ after LPA injection and LPA co-injection with Y27632, compared with the control group $(\mathrm{P}<0.05)$ (Fig. 6B-E). However, the mRNA and protein expression levels of MMP-9 and uPA were significantly lower at $24 \mathrm{~h}$ after LPA co-injection with Y27632 compared with that at $24 \mathrm{~h}$ after LPA injection $(\mathrm{P}<0.05)$ (Fig. 6B-E).

\section{Discussion}

In the present study, the underlying mechanisms by which LPA increases the permeability of BBB have been demonstrated. LPA was found to increase BBB permeability through the expression of proteolytic enzymes, MMP-9 and uPA. Moreover, we have provided evidence that ROCK is involved in LPA-induced proteolytic enzyme expression and $\mathrm{BBB}$ disruption. These findings suggest a crucial role of LPA in BBB damage, which may be due to activation of the Rho/ROCK pathway and upregulation of the important proteolytic enzymes MMP-9 and uPA.

The basement membrane is one of the basic components of the BBB. Protein and collagen that compose the basement membrane can be degraded by a variety of extracellular 
A

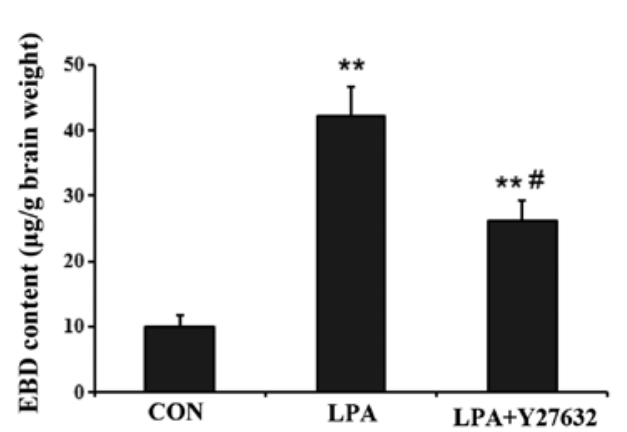

B

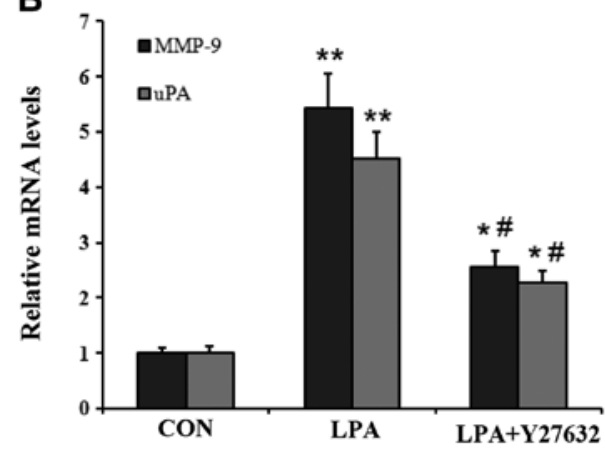

C

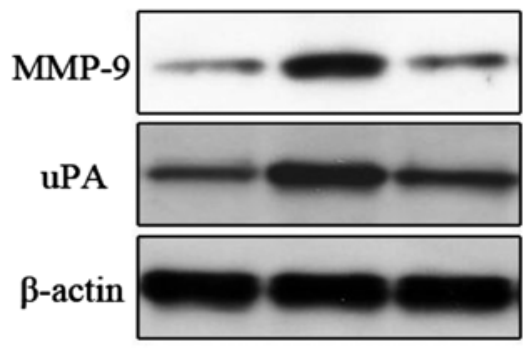

D

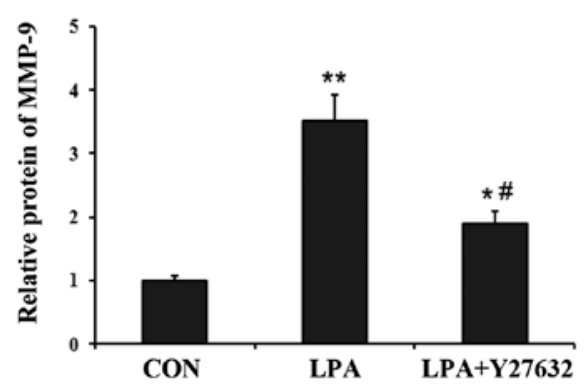

E

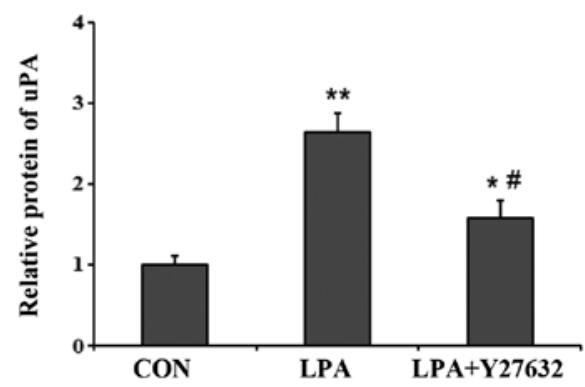

Figure 6. Decreased blood-brain barrier (BBB) permeability and inhibited proteolytic enzyme expression by Rho/Rho kinase (ROCK) inhibitor Y27632. (A) The Evans blue dye (EBD) content in the ipsilateral striatum at $24 \mathrm{~h}$ after lysophosphatidic acid (LPA) injection and LPA co-injection with Y27632 and controls. (B) The mRNA expression of matrix metalloproteinase (MMP)-9 and urokinase-type plasminogen activator (uPA) in the ipsilateral striatum at $24 \mathrm{~h}$ after LPA injection and LPA co-injection with Y27632 and controls. Relative mRNA levels of MMP-9 and uPA normalized to glyceraldehyde 3-phosphate dehydrogenase (GAPDH) and compared with controls. (C-E) A representative western blot and quantitative results for MMP-9 and uPA protein expression in the ipsilateral striatum are shown for the analysis at $24 \mathrm{~h}$ after LPA injection and LPA co-injection with Y27632 and the controls. The relative protein levels of MMP-9 and uPA were normalized to $\beta$-actin and compared with the controls. ${ }^{*} \mathrm{P}<0.05$ and ${ }^{* *} \mathrm{P}<0.01$ vs. the control group; ${ }^{\#} \mathrm{P}<0.05$ vs. the LPA group.

proteolytic enzymes, including the MMP family. MMPs play a critical role in the degradation of neurovascular integrity with BBB disruption. MMPs comprise a large family of zinc-endopeptidases that increase the permeability of BBB by degrading the extracellular matrix and tight junction proteins in endothelial cells (19). MMPs, particularly MMP-9, have a critical function in the proteolytic degradation of the basement membrane of BBB in the pathological process $(20,21)$. Early appearance of the activated MMP-9 has been associated with an alteration of the BBB permeability (22) and the formation of vasogenic edema (23) subsequent to ischemic stroke (24-26) and $\mathrm{ICH}(27,28)$. uPA is the serine protease that converts plasminogen to the active protease plasmin. In addition to playing a role in fibrinolysis, uPA may damage the basement membrane of BBB via proteolysis and extracellular matrix breakdown. The increased expression of uPA is detected in the ischemic cerebral tissues and posttraumatic brain (29-31), which may be responsible for the breakdown of the BBB (32). In the present study, the results have shown that MMP-9 and uPA were increased in the mRNA and protein level in the LPA-injected striatum, which was parallel to EBD leakage, suggesting that LPA induced the expression of proteolytic enzymes MMP-9 and UPA that caused BBB dysfunction.

In studies on tumor cell progression and invasion, LPA has been found to upregulate the expression of MMP-9 and uPA through the Rho/ROCK signaling pathway $(15,33)$. ROCK is a well-known downstream effector of Rho and plays an important role in various physiopathological processes. For example, ROCK is involved in vascular smooth muscle cell contraction, actin cytoskeleton organization, cell adhesion and motility. Accumulating data support a critical role of the Rho/ROCK pathway in increasing permeability of $\operatorname{BBB}(12,13)$. The possible mechanisms are the disruption of tight junction proteins occluding and zonula occludens-1 (13) and the breakdown of basal membrane by the upregulation of MMP-9 and downregulation of laminin expression (12).

Although recent findings suggest that Rho/ROCK pathway is crucial in LPA-induced tumor progression $(15,34)$, the definite mechanism by which Rho/ROCK pathway mediates the breakdown of the BBB induced by LPA remains unclear. 
In this study, we found a similar pattern of changes in the ROCK2 expression, the brain EBD content that indicated BBB permeability, and the expression of MMP-9 and UPA in the ipsilateral striatum at different time-points following LPA intracerebral injection. The present results support the hypothesis that upregulation of the ROCK 2 induced by LPA contributes to the increase of proteolytic enzymes and subsequently BBB dysfunction. On the one hand, LPA induced the expression of ROCK 2 and stimulated the synthesis of proteolytic enzymes leading to the increase of BBB permeability. On the other hand, the inhibitor of ROCK2, Y27632, blocked these subsequent actions induced by LPA in the right striatum. Thus, we have demonstrated that the Rho/ROCK pathway may be involved in LPA-induced proteolytic enzyme expression and $\mathrm{BBB}$ disruption.

Jeong et al (15) reported that the Rho/ROCK pathway, as a critical mediator, connected Ras to $\mathrm{NF}-\kappa \mathrm{B}$ in LPA-induced proteolytic enzyme secretion in ovarian cancer cells, and that a Gi-selective inhibitor pertussis toxin significantly inhibited LPA-induced Rho activation, supporting the important role of $\mathrm{Gi}$ in Ras and Rho activation in the LPA signaling axis. Although little is known regarding the mechanism that the Rho/ROCK pathway mediates BBB dysfunction induced by LPA, the importance of destructive effects of LPA on the central nervous system in various pathological conditions cannot be ignored. Therefore, the explicit mechanism by which LPA induces BBB disruption through the Rho/ROCK pathway remains to be confirmed in future studies by engaging the LPA receptor antagonist and other antagonists in this signaling cascade.

In conclusion, results of the present study have shown that LPA increases BBB permeability by upregulation of proteolytic enzymes MMP-9 and uPA, which may be suppressed by the ROCK inhibitor. The present results suggest that LPA induces BBB disruption through the Rho/ROCK signaling pathway and subsequent production of proteolytic enzymes MMP-9 and UPA. However, future studies are needed to confirm the explicit mechanism by which LPA induces the BBB dysfunction through the Rho/ROCK pathway.

\section{Acknowledgements}

This study was supported by grants from the National Natural Science Foundation of China (81300984), the Natural Science Foundation of Huber Province of China (no. 2010CHB02001) and the Innovation Seed Fund of Wuhan University School of Medicine.

\section{References}

1. Moolenaar WH: Bioactive lysophospholipids and their G protein-coupled receptors. Exp Cell Res 253: 230-238, 1999.

2. Van Meeteren LA, Frederiks F, Giepmans BN, et al: Spider and bacterial sphingomyelinases D target cellular lysophosphatidic acid receptors by hydrolyzing lysophosphatidylcholine. J Bio Chem 279: 10833-10836, 2004.

3. Tokumura A: Metabolic pathways and physiological and pathological significances of lysolipid phosphate mediators. J Cell Biochem 92: 869-881, 2004.

4. Sorensen SD, Nicole O, Peavy RD, et al: Common signaling pathways link activation of murine PAR-1, LPA, and S1P receptors to proliferation of astrocytes. Mol Pharmacol 64: 1199-1209, 2003.

5. Tigyi G and Parrill AL: Molecular mechanisms of lysophosphatidic acid action. Prog Lipid Res 42: 498-526, 2003.
6. Inoue M, Xie W, Matsushita Y, Chun J, Aoki J and Ueda H: Lysophosphatidylcholine induces neuropathic pain through an action of autotaxin to generate lysophosphatidic acid. Neuroscience 152: 296-298, 2008

7. Mauco G, Chap H, Simon MF and Douste-Blazy L: Phosphatidic and lysophosphatidic acid production in phospholipase $\mathrm{C}$-and thrombin-treated platelets. Possible involvement of a platelet lipase. Biochimie 60: 653-661, 1978.

8. Tigyi G, Hong L, Yakubu M, Parfenova H, Shibata M and Leffler CW: Lysophosphatidic acid alters cerebrovascular reactivity in piglets. Am J Physiol 268: H2048-H2055, 1995.

9. Sun GY, Lu FL, Lin SE and Ko MR: Decapitation ischemia-induced release of free fatty acids in mouse brain. Relationship with diacylglycerols and lysophospholipids. Mol Chem Neuropathol 17: 39-50, 1992.

10. Chen KM, Liu JY, Lai SC, Hsu LS and Lee HH: Association of plasminogen activators and matrix metalloproteinase-9 proteolytic cascade with blood-CNS barrier damage of angiostrongyliasis. Int J Exp Pathol 87: 113-119, 2006.

11. Schulze C, Smales C, Rubin LLand Staddon JM: Lysophosphatidic acid increases tight junction permeability in cultured brain endothelial cells. J Neurochem 68: 991-1000, 1997.

12. Fujii M, Duris K, Altay O, Soejima Y, Sherchan P and Zhang JH: Inhibition of Rho kinase by hydroxyfasudil attenuates brain edema after subarachnoid hemorrhage in rats. Neurochem Int 60: 327-333, 2012.

13. Liu K, Li Z, Wu T and Ding S: Role of rho kinase in microvascular damage following cerebral ischemia reperfusion in rats. Int J Mol Sci 12: 1222-1231, 2011.

14. Ishiguro M, Kawasaki K, Suzuki Y, et al: A Rho kinase (ROCK) inhibitor, fasudil, prevents matrix metalloproteinase-9-related hemorrhagic transformation in mice treated with tissue plasminogen activator. Neuroscience 220: 302-312, 2012.

15. Jeong KJ, Park SY, Cho KH, et al: The Rho/ROCK pathway for lysophosphatidic acid-induced proteolytic enzyme expression and ovarian cancer cell invasion. Oncogene 31: 4279-4289, 2012.

16. Baskaya MK, Dogan A, Rao AM and Dempsey RJ: Neuroprotective effects of citicoline on brain edema and blood-brain barrier breakdown after traumatic brain injury. J Neurosurg 92: 448-452, 2000

17. Tawara S and Shimokawa H: Progress of the study of rho-kinase and future perspective of the inhibitor. Yakugaku Zasshi 127: 501-514, 2007.

18. Huang XN, Fu J and Wang WZ: The effects of fasudil on the permeability of the rat blood-brain barrier and blood-spinal cord barrier following experimental autoimmune encephalomyelitis. J Neuroimmunol 239: 61-67, 2011.

19. Rosenberg GA: Matrix metalloproteinases and their multiple roles in neurodegenerative diseases. Lancet Neurol 8: 205-216, 2009.

20. Asahi M, Asahi K, Jung JC, del Zoppo GJ, Fini ME and Lo EH: Role for matrix metalloproteinase 9 after focal cerebral ischemia: effects of gene knockout and enzyme inhibition with BB-94. J Cereb Blood Flow Metab 20: 1681-1689, 2000.

21. Asahi M, Wang X, Mori T, et al: Effects of matrix metalloproteinase-9 gene knock-out on the proteolysis of blood-brain barrier and white matter components after cerebral ischemia. J Neurosci 21: 7724-7732, 2001.

22. Barr TL, Latour LL, Lee KY, et al: Blood-brain barrier disruption in humans is independently associated with increased matrix metalloproteinase-9. Stroke 41: e123-e128, 2010.

23. Rosell A,CuadradoE, Ortega-Aznar A,Hernandez-Guillamon M, Lo EH and Montaner J: MMP-9-positive neutrophil infiltration is associated to blood-brain barrier breakdown and basal lamina type IV collagen degradation during hemorrhagic transformation after human ischemic stroke. Stroke 39: 1121-1126, 2008.

24. Romanic AM, White RF, Arleth AJ, Ohlstein EH and Barone FC: Matrix metalloproteinase expression increases after cerebral focal ischemia in rats: inhibition of matrix metalloproteinase- 9 reduces infarct size. Stroke 29: 1020-1030, 1998.

25. Gasche Y, Fujimura M, Morita-Fujimura Y, Copin JC, Kawase M, Massengale $\mathbf{J}$ and Chan PH: Early appearance of activated matrix metalloproteinase-9 after focal cerebral ischemia in mice: a possible role in blood-brain barrier dysfunction. J Cereb Blood Flow Metab 19: 1020-1028, 1999.

26. Aoki T, Sumii T, Mori T, Wang X and Lo EH: Blood-brain barrier disruption and matrix metalloproteinase-9 expression during reperfusion injury: mechanical versus embolic focal ischemia in spontaneously hypertensive rats. Stroke 33: 2711-2717, 2002. 
27. Wang $\mathbf{J}$ and Tsirka SE: Neuroprotection by inhibition of matrix metalloproteinases in a mouse model of intracerebral haemorrhage. Brain 128: 1622-1633, 2005.

28. Wu H, Zhang Z, Li Y, et al: Time course of upregulation of inflammatory mediators in the hemorrhagic brain in rats: correlation with brain edema. Neurochem Int 57: 248-253, 2010.

29. Dietzmann K, von Bossanyi P, Krause D, Wittig H, Mawrin C and Kirches E: Expression of the plasminogen activator system and the inhibitors PAI-1 and PAI-2 in posttraumatic lesions of the CNS and brain injuries following dramatic circulatory arrests: an immunohistochemical study. Pathol Res Pract 196: 15-21, 2000.

30. Ito T, Takenaka K, Sakai H, Yoshimura S, Hayashi K, Noda S and Sakai N: Elevation of mRNA levels of tissue-type plasminogen activator and urokinase-type plasminogen activator in hippocampus and cerebral cortex following middle cerebral artery occlusion in rats. Neurol Res 22: 413-419, 2000.
31. Armstead WM, Cines DB, Bdeir K, Kulikovskaya I, Stein SC and Higazi AA: uPA impairs cerebrovasodilation after hypoxia/ischemia through LRP and ERK MAPK. Brain Res 1231: 121-131, 2008.

32. Patel TH, Sprague S, Lai Q, Jimenez DF, Barone CM and Ding Y: Blood brain barrier (BBB) dysfunction associated with increased expression of tissue and urokinase plasminogen activators following peripheral thermal injury. Neurosci Lett 444: 222-226, 2008.

33. Park SY, Jeong KJ, Panupinthu N, et al: Lysophosphatidic acid augments human hepatocellular carcinoma cell invasion through LPA1 receptor and MMP-9 expression. Oncogene 30: 1351-1359, 2011.

34. Sawada K, Morishige K, Tahara M, Ikebuchi Y, Kawagishi R, Tasaka K and Murata Y: Lysophosphatidic acid induces focal adhesion assembly through Rho/Rho-associated kinase pathway in human ovarian cancer cells. Gynecol Oncol 87: 252-259, 2002. 